\title{
Konventionelle und neve Kohlenstofformen in ihrer modernen Anwendung
}

\author{
Asta Richter, Ronald Ries, Heiner Vollstädt und Heidrun Recht
}

Diamanten kommen in der Natur vor und werden technisch über die Hochdruck-Hochtemperatur-Synthese erzeugt oder als polykristalline Schicht durch chemische Gasphasenabscheidung aus einem Kohlenwasserstoffplasma abgeschieden. Mit der Verarbeitung von Diamantkörnern in Verbundwerkstoffen werden neue leistungsfähige Schneid- und Polierwerkzeuge entwikkelt. Diamantbeschichtungen oder Diamantkeramiken ermöglichen die Herstellung von dünnen Schneidwerkzeugen und Anwendungen in Elektronik und Optik mit extremer Belastbarkeit. Fullerene, Nanoröhrchen und Zwiebelstrukturen aus Kohlenstoff haben sich seit ihrer Entdeckung im Jahre 1985 zu einem eigenständigen Forschungsgebiet entwickelt und besitzen außergewöhnliche mechanische, optische und elektronische Eigenschaften, die sie für unterschiedlichste Anwendungen prädestinieren.

\section{Kohlenstoff ist ein besonderes Atom}

Die geringe Ausdehnung des Kohlenstoffatoms und seine vier Valenzelektronen führen zu einer Vielfalt von Bindungsmöglichkeiten, wie sie für kein anderes Atom bekannt sind. Dies hat auch Konsequenzen für die Materialwissenschaft. So enthalten alle Polymere Kohlenstoffatome. Trotz des grundsätzlichen Wissens über die interessanten Bindungsverhältnisse, ist man immer wieder überrascht, welche ausgefallenen Strukturen sich aus diesem Atom herstellen lassen. So kennen wir heute von den reinen Kohlenstoffmodifikationen nicht nur Diamant und Graphit, sondern seit 1985 auch das Fußballmolekül $\mathrm{C}_{60}$, weitere Fullerene, Nanoröhrchen und Kohlenstoffzwiebeln [1].

Diamanten gehören zu den schönsten und interessantesten Materialien, die wir kennen, und besitzen viele praktische und industrielle Anwendungen [2]. Sie sind mit einer Härte von ca. $90 \mathrm{GPa}$ der härteste derzeit bekannte Werkstoff. Chemische Resistenz, Biokompatibilität und ein kleiner Reibungskoeffizient sind weitere nuitzliche Eigenschaften für medizinische und tribologische Anwendungen. Die optische Transparenz im weiten Bereich von Ultraviolett bei $230 \mathrm{~nm}$ bis zum Infrarot bei $2,5 \mu \mathrm{m}$, eine optische Brechzahl von 2,417 und eine hohe Wärmeleitfähigkeit, die etwa sechsmal größer als die von Kupfer ist, führen zu Applikationen als Schutzschichten in Linsensystemen, Masken fuir die Röntgenlithographie und Anwendungen bei der Lösung von Problemen mit höchsten thermischen Leistungsdichten. Der Werkstoff Diamant besitzt auch außergewöhnliche elektronische Eigenschaften. Dazu gehören eine große elektronische Bandluicke von $5,5 \mathrm{eV}$, eine hohe Ladungsträgerbeweglichkeit und eine hohe Driftgeschwindigkeit. Dies könnte zu neuartigen Bauelementen für elektronische Geräte beim Einsatz unter hohen Temperaturen und hohen Frequenzen fuihren.

\section{Einkristalliner Diamant}

Diamanten werden in der Natur gefunden und dort abgebaut. Sie werden auch synthetisch hergestellt. Die größten Diamantkristalle kommen als natürliche Diamanten in der Erdkruste vor. Kleinere Körner von einigen Millimetern Durchmesser werden über die Hochdruck-Hochtemperatur Diamantsynthese aus Graphit hergestellt. Polykristalline Diamantschichten bilden sich auf einem Substrat durch chemische Gasphasenabscheidung aus einem Kohlenwasserstoffplasma. Bei der Untersuchung dieser Stoffe geht es darum, den Mechanismus der Diamantbildung zu verstehen und gezielt durch die technologischen Verfahren zu beeinflussen, um Qualität und Größe der synthetischen Diamantkörner zu verbessern.

Industriell werden Diamantkörner als super-hartes Material in Diamantwerkzeugen zum Bohren, Schneiden und Polieren von harten Materialien, z. B. zum Schneiden von Hartstoffen, Halbleiter-Wafern und Edelsteinen, aber auch für Trennarbeiten im Straßenbau eingesetzt [2]. Viele Härteprïfgeräte arbeiten mit Diamantprüfkörpern. Aus einkristallinen Diamanten werden auch hochwertige, besonders elegante und teure Schmuckstiicke gefertigt.

Diamanten bestehen aus Kohlenstoffatomen, die im kubische Kristallgitter periodisch angeordnet sind. Die Kohlenstoffatome sitzen auf den Plätzen von zwei flächenzentrierten Würfelgittern, die entlang der Raumdiagonale um 1/4 versetzt angeordnet sind. Dadurch ergibt sich eine tetraedrische Koordination zwischen den Atomen im Inneren des Würfels mit denen auf den Würfelkanten und auf den Würfelflächen. Typische Kristallwachstumsformen dieser atomaren Anordnung sind, Oktaeder, Würfel, Rhombendodekaeder und KuboOktaeder, eine Kombination aus Würfel und Oktaeder. In der Abbildung 1 sind einige dieser Wachstumsformen von natïrlichen Diamanten zu sehen, die mit dem Rasterelektronenmikroskop gewonnen wurden. Neben typischen, glatten Oktaederformen ist die zerkluiftete und rauhe Oberfläche eines Kubo-Oktaeders sichtbar. Dies dokumentiert die unterschiedlichen Wachstumsgeschwindigkeiten der (111) und (100) Flächen. Die 

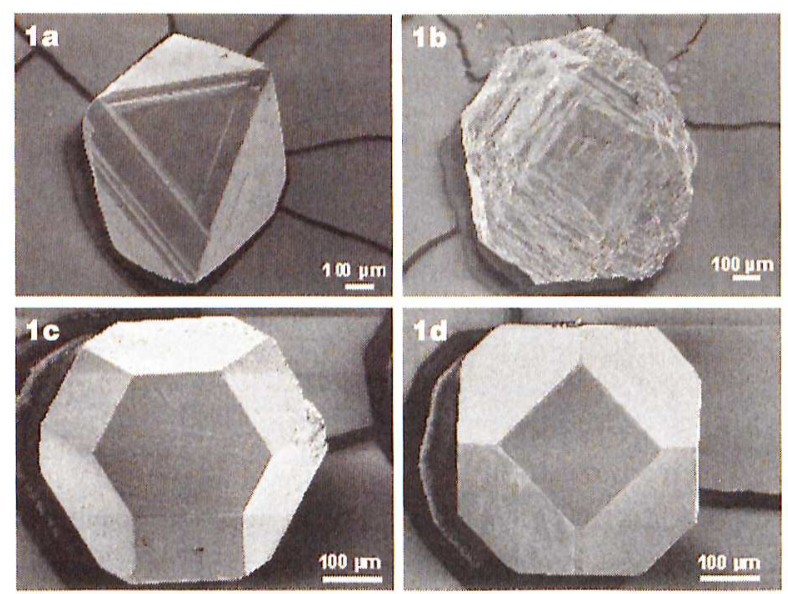

Abb. 1: Rasterelektronenmikroskopie-Bilder von Rohdiamanten $(a, b)$ und synthetischen Diamanten der Firma DeBeers (c, d); Oktaeder mit typischer (111) Wachstumsfläche (a), Kubo-Oktaeder mit dominanter (100) Fläche (b), Kubo-Oktaeder mit typischer (111) Fläche (c) und (100) Fläche.

Oktaederkristalle repräsentieren die (111) Wachstumsfläche, die sehr eben und terrassenförmig als dicht gepackte Netzebene angelegt ist. Deutlich sieht man dreieckige Wachstumsstrukturen auf größeren Dreiecksflächen, die ein terrassenförmiges Lagenwachstum charakterisieren.

Die sehr ebenen (111) Flächen eignen sich gut zur Abbildung mit dem Rasterkraftmikroskop (SFM) [3]. Mit dem SFM können hochauflösende Abbildungen der Probenoberfläche an Luft ohne zusätzliche Präparation erhalten werden. Hiermit lassen sich Details der Terrassenstufen abbilden und ausmessen. Manchmal sind die Flächen nicht vollständig gefüllt. Dann bleiben dreieckige Gruben sichtbar (Abb. 2). Das Profil der Grube weist annähernd einen $90^{\circ}$ Winkel auf, der charakteristisch für eine dreiseitige Diamantpyramide ist, die aus (100) Flächen gebildet wird. Es wurden Gruben gefunden, die in der Grubenmitte kein besonderes Merkmal aufwiesen, und Gruben, die eine Erhebung zeigten. Die
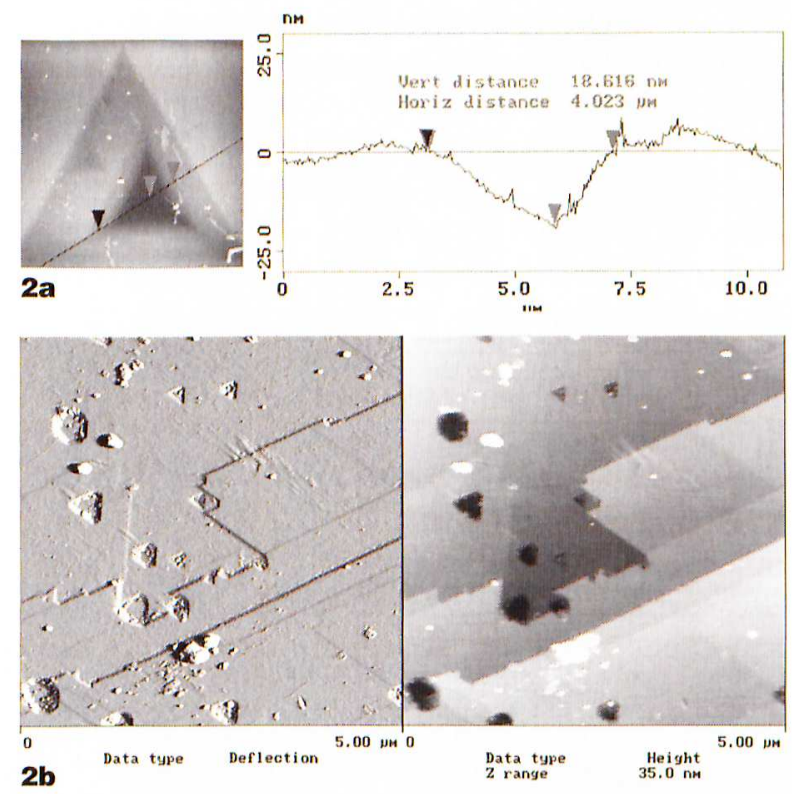

Abb. 2: Rasterkraftmikroskopie an (111) Wachstumsflächen von natürlichem (a) und synthetischem (b) Diamant; deutlich sind Wachstumsgruben und Terrassenwachstum erkennbar:
(111) Gruben können durch Wachstumsprozesse, aber auch durch Ätzung während des Wachstums entstanden sein. Die Ursache für die Bildung der Gruben ist noch nicht geklärt.

Synthetische Diamantkörner und Diamantpulver werden iiber die Katalysator gesteuerte Diamantsynthese hergestellt. Bei der direkten Umwandlung von Graphit in Diamant sind Druicke von mehr als 130 kbar und Temperaturen von mehr als $2500{ }^{\circ} \mathrm{C}$ nötig. Bei der katalytischen Kohlenstoffumwandlung von Graphit in Diamant erfolgt zunächst eine katalytische Graphitisierung, dann eine Lösung des Kohlenstoffs in der Metallschmelze, aus der durch Keimbildung und Keimwachstum Diamantkörner gebildet werden. Druck und Temperatur können auf 50 - 80 kbar bzw. $1200-1800{ }^{\circ} \mathrm{C}$ abgesenkt werden [2]. Als Katalysatoren werden Übergangsmetalle wie Eisen, Kobalt, Nickel, Mangan, Chrom, Tantal, Niob oder andere Stoffe elementar oder in Kombination eingesetzt. Die Katalysatoren sollten eine gute Benetzbarkeit, Bindungslöslichkeit und eine gute Festlöslichkeit für Kohlenstoff besitzen. Sie wirken als Lösungsmittel, wobei die Löslichkeit von Graphit größer als die von Diamant ist. Bei einem bestimmten Temperaturgradienten kristallisieren Diamantkeime aus dieser Lösung aus. Der Lösungskatalysator kann auch Zwischenprodukte mit Kohlenstoff bilden, wie z. B. Karbide oder Mischkristalle, die ebenfalls günstig für die Diamantsynthese sind. Bei der Keimbildung der Diamanten sollte der Katalysator eine Wellung der Kohlenstoffschichten bewirken und in das Graphitgitter diffundieren.

Lichtmikroskopischen Aufnahmen von synthetischen Diamantkristallen im Durchlichtverfahren zeigen dunkle Stellen, die Ausscheidungen von Graphit darstellen, die Katalysatormaterial enthalten. Diese Ausscheidungen beeinträchtigen die Qualität der synthetischen Diamantkörner. Die Katalysatorspuren können deutlich in den SFM-Aufnahmen (Abb. 2, 3) von synthetischen Diamantoberflächen analysiert werden. Wie auch bei natürlichen Diamanten sind die (111) Flächen bei synthetischen Diamanten ebener als die (100) Flächen. Die Terrassenstruktur mit den typischen (111) Gruben ist ebenfalls sichtbar, wenngleich nicht so ausgeprägt wie bei den natuirlichen Diamanten. Die Terrassenstufen sind mit minimal 2,3 nm auch höher als bei natürlichen Diamanten. Neben den (111) Wachstumsgruben wurden andere Hohlformen als Gräben gefunden, in denen sich Katalysatorrüickstände sammeln können. Die (100) Wachstumsflächen von synthetischen Diamanten zeigen parallel liegende Wachstums-terrassen oder Dendritenwachstum mit Diffusionskanälen. Die Wachstumsterrassen haben eine Breite von $1-2 \mu \mathrm{m}$, während die Diffusionskanäle mit ca. $40 \mathrm{~nm}$ wesentlich schmaler sind. Die Diffusionskanäle liegen etwas tiefer wie Gräben zwischen den Terrassen. Zweidimensionales Dendritenwachstum ist auf den (100) Wachstumsflächen ebenfalls sichtbar. Die Dendritenstruktur ist von einem feinen Diffusionskanal umgeben. In den Diffusionskanälen befinden sich graphitische Anteile und Katalysatormaterial. Hier zeigt sich in der morphologischen Struktur ein Ansatz zur Verbesserung der Diamantsynthese und zur Qualitätskontrolle der Diamantkörner. 

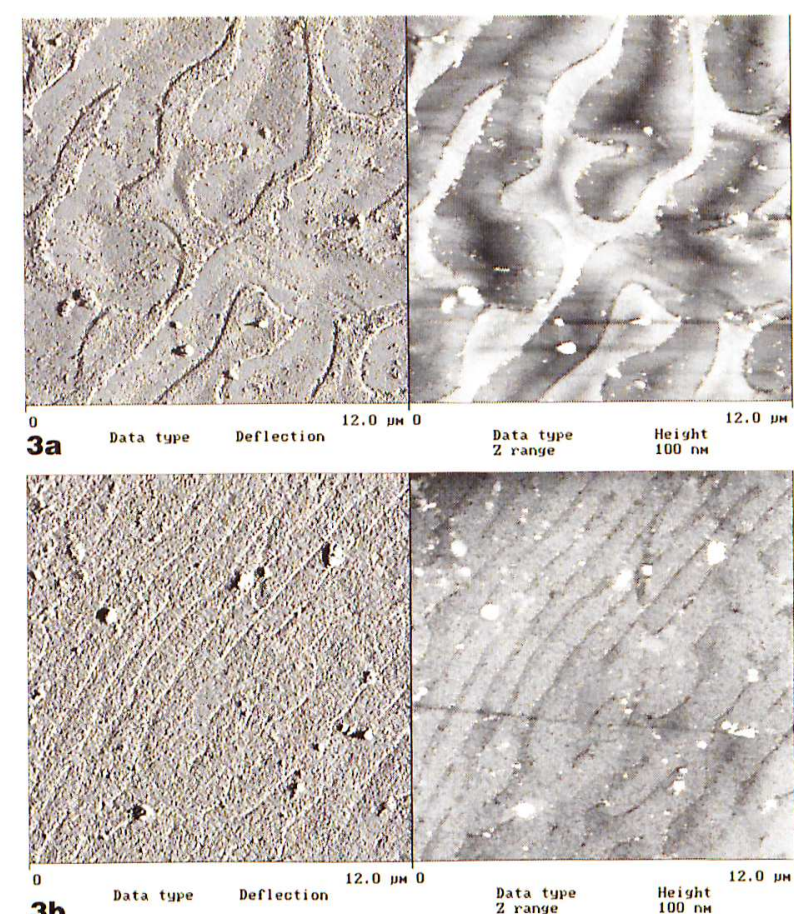

3b

Data type
2 range

Height

Abb. 3: Rasterkraftmikroskopie an einer (100) Wachstumsfläche von synthetischen Diamanteinkristallen; Dendritenwachstum mit Diffusionskanälen (a) und Wachstumsterrassen mit parallelen Diffusionskanälen (b).

Mit einem Nanoindenter der Firma Hysitron, der mit einer dreiseitige Diamantpyramide mit $90^{\circ}$ Öffnungswinkel an einem Stahlcantilever betrieben wird [4], wurde die Härte der synthetischen Diamantkristalle geprïft. Die Diamantpyramide wird von den (100) Flächen der Diamantstruktur begrenzt und stellt damit den härtesten Prüfkörper dar, der gegenwärtig verfügbar ist. Erwartungsgemäß kann der Diamantprüfkörper keinen plastischen Eindruck in den synthetischen Diamanten erzeugen. Die Kraft-Eindringkurve zeigt jedoch einen typischen elastischen Bereich, aus dem sowohl die EModul-Werte als auch die Härtewerte berechnet werden können. Für die Nanoindent-Messungen wurden die kleinen Kristalle mit Silber-Leitlack auf einer SFM-Unterlage fixiert. Es wurde mit Kräften bis zu 3,3 mN für die Wachstumsrichtungen (111) und (100) gearbeitet. Die

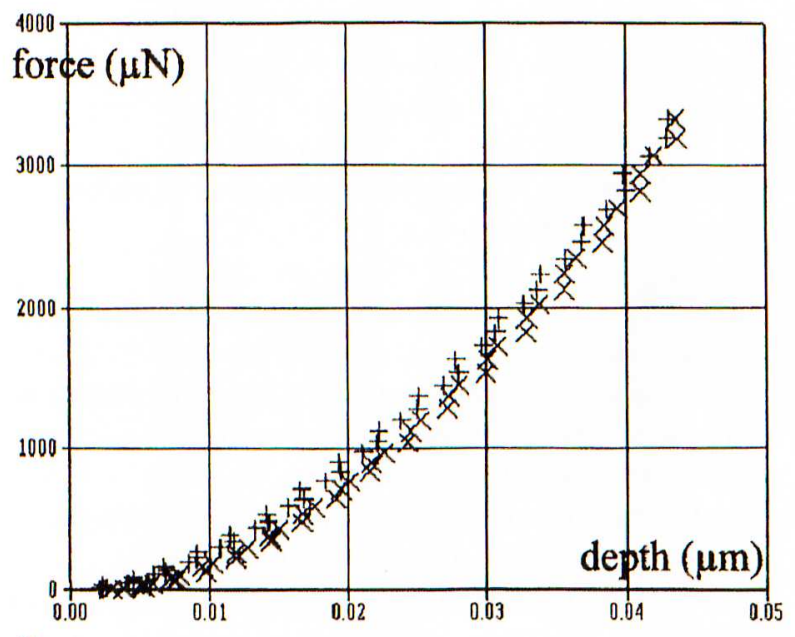

$4 \mathbf{a}$

Abb. 4: Kraft-Eindring-Kurven mit dem Nanoindenter von Hysitron an (100) Wachstumsflächen (a) und (111) Wachstumsflächen (b) von synthetischem Diamant.

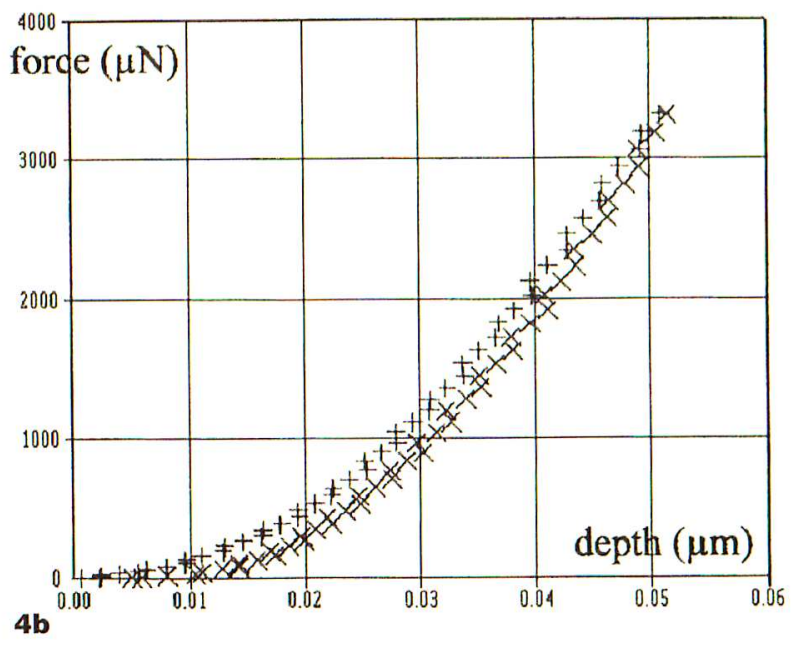

Kraft-Eindring-Kurven sind in Abbildung 4 zu sehen. Aus dem Anstieg der Kurven kann für die beiden Wachstumsrichtungen (111) und (100) der E-Modul und die Universal-Härte (HU) ermittelt werden. Die Anisotropie der mechanischen Eigenschaften für unterschiedliche Kristallrichtungen kommt deutlich zum Ausdruck: E(111) $=1166 \mathrm{GPa}$ und $\mathrm{E}(100)=1014 \mathrm{GPa}$ sowie $\mathrm{HU}(111)=$ $134 \mathrm{GPa}$ und $\mathrm{HU}(100)=116 \mathrm{GPa}$. Diese Werte für den EModul und die Universalhärte liegen im Bereich der Härteangaben für Diamant aus der Literatur [5]. Entsprechend der Dichte der kristallographischen Netzebenen ist der Härtewert der (111) Fläche etwas größer als der der (100) Wachstumsfläche.

\section{Diamantschichten}

In den 80iger Jahren etablierte sich ein Nicht-Gleichgewichts-Verfahren zur Abscheidung von polykristallinen Diamantschichten aus einem Gasgemisch von Kohlenwasserstoffen (z. B. Methan) und Wasserstoff. Durch ein Mikrowellenplasma werden die Gasmoleküle angeregt und kondensieren im Rahmen einer chemischen Gasphasenabscheidung (CVD) unter Wirkung von heißen Metallfilamenten auf Heterosubstraten zu Diamantfilmen [6]. Dies ist ein Niedertemperatur-NiederdruckProzeß zur Beschichtung von großen Flächen bei hohen Abscheideraten. Der hochkomplexe Wachstumsprozeß ist im Detail noch nicht vollständig verstanden, wird aber in Hochtechnologie-Firmen und auch klein- und mittelständischen Unternehmen bereits industriell durchgeführt.

Mit den CVD-Diamanten werden neue Einsatzfelder abgedeckt, die mit Natur- oder synthetischen Diamanteinkristallen nicht zu realisieren sind. Dünne Schneidwerkzeuge für hoch präzise Schneidprozesse (Tab. 1) weisen eine Vervielfachung der Standzeiten und eine Minimierung des Schneidverlustes auf. Hochgeschwindigkeitstrennen von Hartstoffen ohne Kuihlmittel wird ebenfalls mit Diamantschichten möglich. Wesentlicher Vorteil der Diamantschicht-Verbunde ist jedoch, daß kein Bindemittel notwendig ist, um die Diamantkörner einzubetten. Der Abrieb wird allein durch den Verschleiß der Diamantschicht bestimmt, der Diamantgehalt der Schicht beträgt $100 \%$. Die Trennwärme kann zum Schmelzen des Bindemittels führen, wohingegen die 
Tabelle 1: Anwendungsbeispiel dünne Schneidwerkzeuge und weitere Applikationen von Diamantschichten

\begin{tabular}{|c|c|c|c|}
\hline Anwendung & Vorteil / Kundennutzen & Vorteil & Verbesserung für folgende Spezifikation(en) im Prozeß \\
\hline Schneiden von Hartstoffen & $\begin{array}{l}\text { - Vervielfältigung der Standzeit } \\
\text { - stabilere Schnittgeometrie }\end{array}$ & $\begin{array}{l}++ \\
+\end{array}$ & Standzeit, Robustheit, Geometrie (Toleranz des Schnittes) \\
\hline $\begin{array}{l}\text { Hochgeschwindigkeitstren- } \\
\text { nen von Hartkeramik }\end{array}$ & $\begin{array}{l}\text { - kein Kühlmittel bei Grün-Bearbeitung erforderlich } \\
\text { - Vervielfachung der Standzeit }\end{array}$ & $\begin{array}{l}+1 \\
+++ \\
+++\end{array}$ & Standzeit, Robustheit, Geometrie \\
\hline Schneiden von Si-Chips & $\begin{array}{l}\text { - Vervielfältigung der Standzeit } \\
\text { - erhöhte Ausbeute durch geringere Schnittverluste } \\
\text { - keine Verunreinigung durch Bindemittel }\end{array}$ & $\begin{array}{l}+++ \\
++ \\
+\end{array}$ & $\begin{array}{l}\text { verringerte Breite des Schnittes } \\
\text { Standzeit, Robustheit, Geometrie (Toleranz) }\end{array}$ \\
\hline $\begin{array}{l}\text { Schneiden von SiC-Wafern } \\
\text { und-Chips }\end{array}$ & $\begin{array}{l}\text { - Vervielfaltigung der Standzeit } \\
\text { - erhöhte Ausbeute durch geringere Schnittverluste } \\
\text { - keine Verunreinigung durch Bindemittel }\end{array}$ & $\begin{array}{l}+++ \\
++ \\
+\end{array}$ & wie Si-Schneiden \\
\hline Schneiden von GaAs-Chips & $\begin{array}{l}\text { - erhöhte Ausbeute durch geringere Schnittverluste } \\
\text { - Verminderung des giftigen Schnittschlammes }\end{array}$ & $\begin{array}{l}++ \\
++\end{array}$ & Geometrie (Toleranz), Reinheit, Breite des Schnittes \\
\hline Trennen von Edelsteinen & $\begin{array}{l}\text { - Vervielfachung der Standzeit } \\
\text { - erhöhte Ausbeute durch geringere Schnittverluste }\end{array}$ & $\begin{array}{l}+++ \\
+++\end{array}$ & $\begin{array}{l}\text { Standzeit } \\
\text { Schnittverlust minimiert durch verringerte Breite des Schnittes }\end{array}$ \\
\hline $\begin{array}{l}\text { Trennen von plastischen } \\
\text { Metallen }\end{array}$ & keine wesentlichen Vorteile & - & \\
\hline
\end{tabular}

Weitere Applikationen von Diamantschichten in anderen technischen Gebieten:

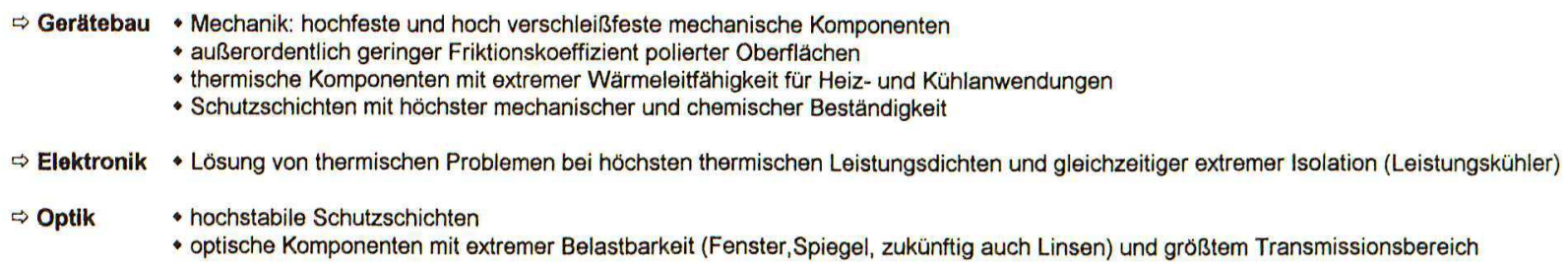

Tabelle 1: Anwendungsbeispiel dünne Schneidwerkzeuge und weitere Applikationen von Diamantschichten.

Wärme bei den Diamantschichten gut abgeleitet wird. Damit ist eine erhöhte Werkzeugtemperatur möglich. Diamantschichten wachsen auch auf keramischen Trägermaterialien auf, die eine geringe Dichte besitzen (Diamantkeramiken). Neben diesen traditionellen Hartstoffanwendungen werden Diamantschichten als optische Komponenten mit extremer Belastbarkeit über einen großen Transmissionsbereich und als temperaturbeständige Hochleistungs-Mikroelektronik-Bauteile verwendet (Tab. 1).

Bei der Herstellung der Diamantschichten ist die Herausbildung einer geeigneten Filmmorphologie wesentlich. Amorphe oder diamantartige Kohlenstoffschichten [7] enthalten immer einen hohen Anteil von graphitischen $\mathrm{sp}^{2}$ Bindungen im Vergleich zu der dreidimensionalen $\mathrm{sp}^{3}$ Bindungen, die typisch für die Diamant-

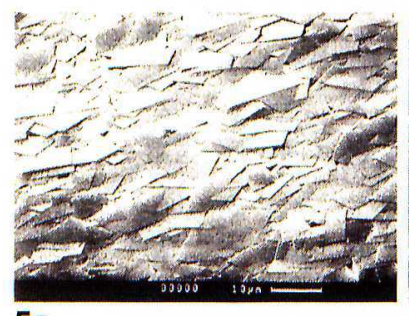

$5 \mathbf{a}$

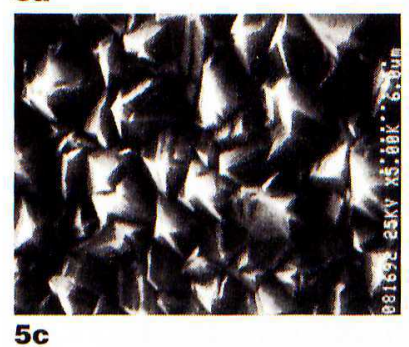

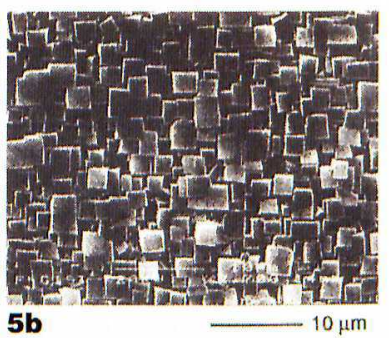

Abb. 5: Rasterelektronenmikroskopie an polykristallinen Diamantfilmen, die aus einem Kohlenwasserstoffplasma mit CVD abgeschieden wurden; typische Oberflächenmorphologien: quadratische (100) Facetten (a), stark texturierte (100) Facetten (b), rauhe, polykristalline Oberfläche mit dreieckigen (111) Flächen (c). bindung sind. Schließt man durch geeignete Prozeßbedingungen $\mathrm{sp}^{2}$ Bindungen aus, so zeigen die polykristallinen Diamantfilme deutlich (100) bzw. (111) Flächenwachstum (Abb. 5), wie es beim Wachstum von Diamanteinkristallen nachgewiesen wurde. Quadratische (100) Facetten können irregulär, aber doch ziemlich flach (Abb. 5a) oder stark texturiert (Abb. 5b) aufwachsen bei geeigneter Steuerung der anisotropen Wachstumsgeschwindigkeiten. Die Herausbildung einer ausgeprägten Textur ist ein wichtiges Detail, um sehr ebene und selbsttragende Diamantschichten für optische und elektronische Anwendungen herzustellen. Texturierte Diamantschichten können mit einer Dicke von 15 bis $60 \mu \mathrm{m}$ bei Rauhigkeiten von weniger als 80 $\mathrm{nm}$ abgeschieden werden. Rauhe Schichtoberflächen mit vielen Korngrenzen, Gitterfehlern und auch (111) Wachstumsflächen sind in Abb. 5c zu sehen.

\section{Fullerene, Nanoröhrchen und Kohlen- stoffzwiebeln}

Seit 1985 gibt es käfigförmige Molekiile aus Kohlenstoff, die Fullerene genannt werden [1].

Das bekannteste Fullerenmolekuil besteht aus 60 Kohlenstoffatomen, das bei einem Durchmesser von $1 \mathrm{~nm}$ wie ein Mini-Fußball (Abb. 6a) aussieht. Diese vollständig synthetischen und sehr stabilen Kohlenstoffkugeln sind neuartige molekulare Materialbausteine. $\mathrm{C}_{60}$-Moleküle bilden feste Stoffe in Form von Einkristallen oder als Fullerenfilme [7]. $\mathrm{C}_{60}$-Moleküle können endohedral mit anderen Atomen gefuillt werden, sie lassen sich linear und dreidimensional zu Polymerstrukturen vernetzen und können dotiert werden. Damit ergibt sich 


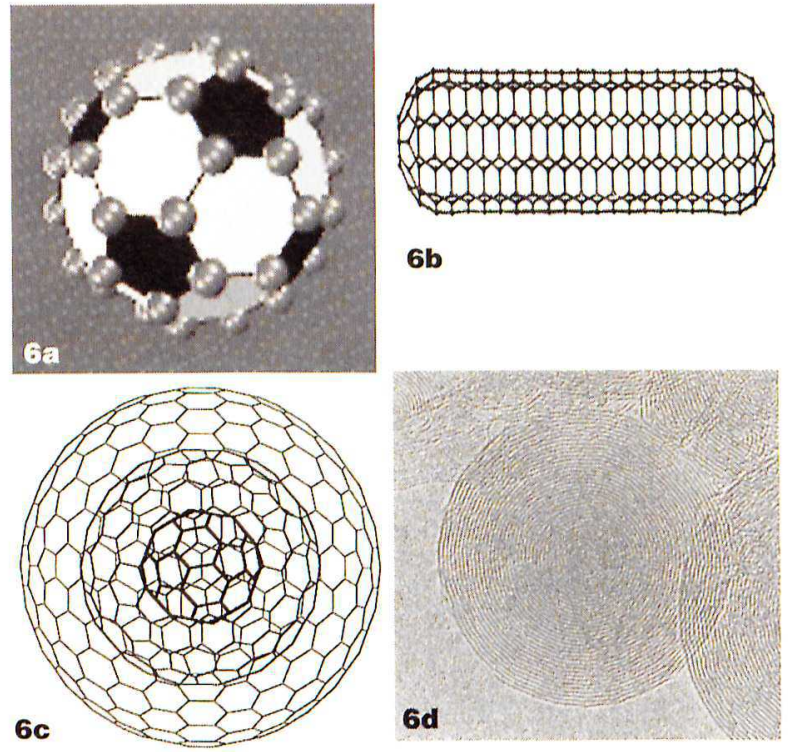

Abb.6: Fullerenmolekül aus 60 Kohlenstoffatomen (a) als Fußballmodell mit 12 Fünfecken und 20 Sechsecken; schematische Darstellung einer Nanoröhre (b); Kohlenstoffzwiebel als Modell (c) und als Abbildung im Transmissionselektronenmikroskop (d).

eine neue Klasse von Materialien, die unter dem Namen Fullerenchemie bekannt ist. Aus $\mathrm{C}_{60}$ Molekuilen lassen sich bei hohen Drüiken auch ultraharte Phasen herstellen [5].

Elektronisch gesehen nimmt $\mathrm{C}_{60}$ eine Zwischenstellung zwischen dem metallischen Graphit und dem isolierenden Diamant ein. Das Molekuil kann auch durch Licht angeregt werden und bis zu sechs zusätzliche Elektronen aufnehmen und wieder abgeben. Damit scheinen Fullerene geeignete Materialien für Ladungsspeicher und photovoltaische Anlagen zu sein. Die gute Kombinierbarkeit dieses Moleküls mit organischen Materialien führte bereits schon zur Patentierung von Solarzellen auf Fullerenbasis [8].

Besondere Bedeutung hat das $\mathrm{C}_{60}$-Molekül für die Herstellung organischer Solarzellen. Konjugierte Polymere mit ausgedehnten p-Elektronensystemen weisen eine elektrische Leitfähigkeit auf. Diese p-Elektronen bilden in einem Polymerkettenmolekuil ein breites HOMO-Band von besetzten Zuständen (Valenzband). Analog gibt es dazu aus den niedrigsten unbesetzten Zuständen ein LUMO-Band (Leitungsband) in dem Kunststoffmoleküil. Durch die quasi-eindimensionale Bandstruktur der halbleitenden Polymerkette gibt es einen wesentlichen Unterschied zu den klassischen anorganischen Halbleitern. Während man in p- und ndotierten anorganischen Halbleitern frei bewegliche delokalisierte Ladungszustände vorfindet, bilden sich in organischen Halbleitern stärker lokalisierte Zustände mit Radikalcharakter aus.

$\mathrm{C}_{60}$-Moleküle können den organischen Halbleitern als Akzeptoren beigemischt werden. Wird ein DonatorPolymermolekuil optisch angeregt, so gelangen Elektronen aus dem HOMO-Band in das LUMO-Band und bilden dort einen lokalisierten Zustand. Befindet sich in der Nähe des Donators ein $C_{60}$-Akzeptor-Molekuil, so erfolgt ein Elektronentransport, der sich so bis zur Kathode fortsetzen kann. Dieser ultraschnelle fotoinduzierte Elektronentransfer an den Grenzflächen zwischen Donator und Akzeptor fuihrt zu einer enormen Steigerung des Wirkungsgrades der organischen Solarzelle [10]. Da der Ladungstransfer nur an den Beriihrungspunkten der Donator- mit den Akzeptormolekuilen stattfinden kann, ist eine Mischung auf molekularer Basis notwendig. Dies wird mit dem $\mathrm{C}_{60}$-Moleküil möglich. Die Ladungstrennung erfolgt durch das Aufbringen elektrischer Kontakte mit unterschiedlichen Austrittsarbeiten. Als Anodenmaterial (Löcher leitende Elektrode) wird vorwiegend ITO (Indium-Zinnoxid) und als Kathodenmaterial (Elektronen leitende Elektrode) Aluminium oder Calcium verwendet (siehe Abb. 7).

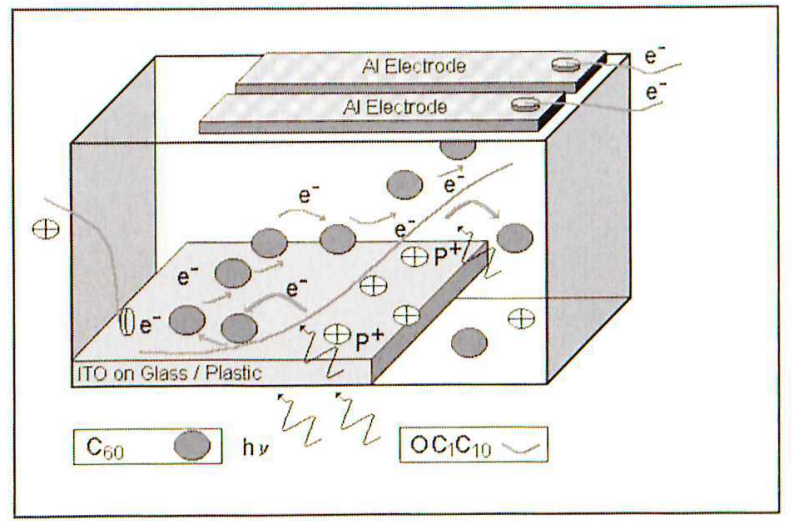

Abb. 7: Schematische Darstellung zum Ladungstransport in einer Solarzelle auf der Basis organischer Materialien.

Obwohl Wirkungsgrad und Langzeitstabilität dieser organischen Solarzelle noch verbessert werden müssen, sind die organischen Polymer/Fulleren-Mischungen interessante Materialien der Zukunft. So wäre es vorstellbar, großflächige Solarzellen auf flexiblen Substraten, z. B. Folien, kostengünstig herzustellen. Dabei könnte die Produktion vollständig ohne die teure Vakuumtechnik auskommen. Sogar Aufsprühprozesse der Polymermischungen sind denkbar.

Weitere Anwendungen des $\mathrm{C}_{60}$-Moleküls sind in Abbildung 8 dargestellt. Dabei sind die Anwendungsbereiche in physikalischen, chemischen und elektro-optischen Eigenschaftsgruppen zusammengefaßt.

Langgezogene Kohlenstoffröhrchen (Abb. 6b) haben Durchmesser von 2-20 nm und können ein- oder mehrwandig sein. Sie werden aus aufgerollten Graphitebenen gebildet. Diese Nanoröhrchen haben eine unglaubliche Elastizität von 4 TPa und besitzen eine besonders hohe Feldemission. Aufgrund dieser Eigenschaften könnten sie zur Verstärkung in polymeren und keramischen Verbundwerkstoffen, aber auch als Nanosensoren für submikroskopische Anwendungen verwendet werden. Sie eignen sich auch als Nanoelektroden und können zur elektronischen Bilderzeugung eingesetzt werden. Die Nanoröhrchen kann man auch füllen und damit neuartige Materialkombinationen erzeugen. Sie eignen sich auch als Wasserstoffspeicher mit einer erstaunlich hohen Speicherkapazität. 


\section{Mögliche Anwendungen von Fullerenen}

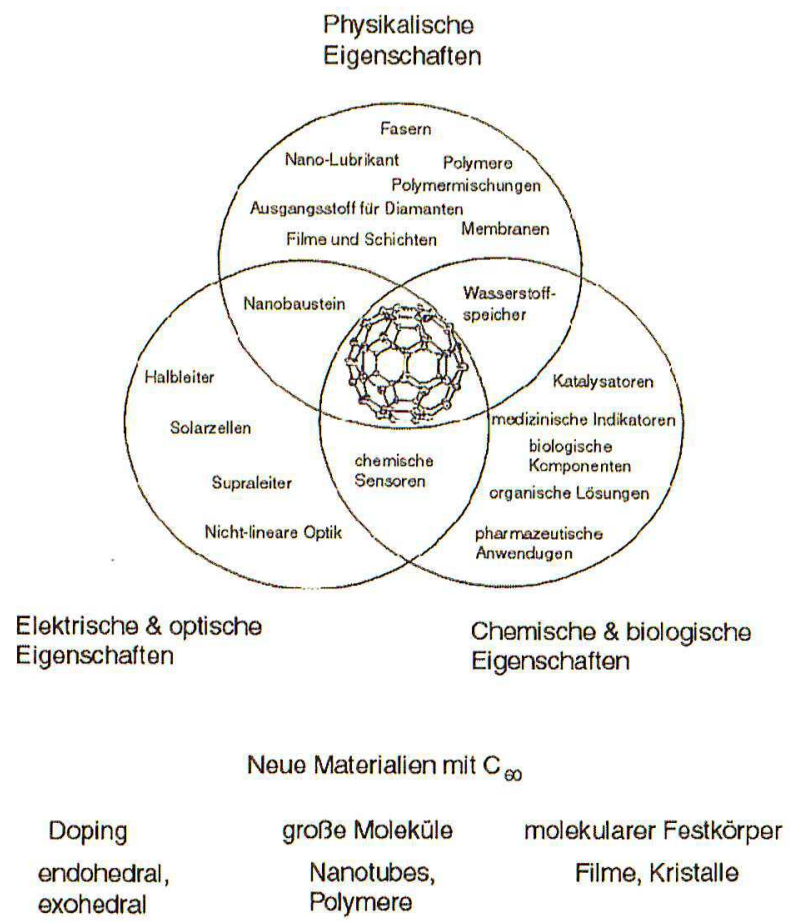

Abb. 8: Mögliche Anwendungen von Fullerenen.

Kohlenstoffzwiebeln sind nanostrukturierte Festkörper mit Durchmessern von einigen $100 \mathrm{~nm}$. Sie entstehen durch Bestrahlung von Ruß mit hochenergetischen Elektronen oder wachsen vereinzelt im Elektronenmikroskop (Abb. 6c und d). Diese Onions stellen eine Sonderform von kugelförmigem Graphit dar und werden aus ineinander geschachtelten Fullerenen gebildet. Die inneren Graphitschalen liegen um etwa ein Drittel näher zusammen, so daß im Inneren der Zwiebel ein immens hoher Druck herrscht. Durch Bestrahlung der Zwiebeln mit Elektronen bilden sich Diamantkeime. Mit dieser Methode wurden bereits $100 \mathrm{~nm}$ große Diamanten erzeugt [11]. Falls sich eines Tages größere Diamanten mit diesem Verfahren herstellen lassen, so sind diese synthetischen Diamanten besonders rein und ohne Ruickstände von Katalysatoren.

Danksagung: Wir danken dem Präsidenten der TFH Wildau, Herrn Prof. Dr. W. Arlt, für das Interesse an der Forschungsthematik und die Unterstuitzung der Arbeiten.

\section{Literatur}

[1] H. Kuzmany, Phys. Bl. 54 (1998) 331-337.

[2] Karl E. Spear and John P. Dismukes, Synthetic Diamond, John Wiley \& Sons New York 1994.

[3] A. Richter and R. Ries, Molecular Physics Reports 21 (1998) 11-20.

[4] A. Richter and R. Ries, SPIE Vol. 3725 / (1999) 94-101.

[5] V.D. Blank, V.N. Denisov, A.N. Ivlev, B.N. Mavrin, N.R. Serebryanaya, G.A. Dubitsky, S.N. Sulyanov, M.Yu. Popov, N.A.Lvova, S.G. Buga and G.N. Kremkova, Carbon 36 (1998) 1263-1267.

[6] R. Sauer, erscheint in Crystal Research and Technology 1999.

[7] A. Richter, R. Ries, K. Szulzewsky, B. Pietzak and R. Smith, Surface Science 394 (1997) 201-220.

[8] A.F. Hebbard, B. Miller, J.M. Rosamilia and W.L. Wilson, USA-Patent 5,171,373 Dec.15,1992.

[9] W. Stieler, c't 2 (1999) 76-81.

[10] Maik Schäfer, Diplomarbeit „Herstellung und Untersuchung organischer Halbleiterstrukturen für solartechnische Anwendungen", TFH Wildau 1999.

[11] F. Banhart, Phys. Bl. 53 (1997) 33-35.

\section{Autoren}

\section{Asta Richter, Ronald Ries}

Technische Fachhochschule Wildau

Physikalische Technik

Bahnhofstraße

15745 Wildau

Heiner Vollstädt, Heidrun Recht

Vollstädt-Diamant GmbH

Schlunkendorfer Straße 21

14554 Seddiner See 